\title{
ARTÍCULOS
}

\section{JUEGO VERBAL, METÁFORA Y SEXUALIDAD. EL DISCURSO DE HUMOR (NAYAKYAGAK) ENTRE LOS TOBAS (QOM) DEL GRAN CHACO SUDAMERICANO*}

\author{
VERBAL PLAY, METAPHOR AND SEXUALITY. NAYAKYAGAK (JOKES) AMONG THE \\ TOBA (QOM) OF THE SOUTH AMERICAN GRAN CHACO REGION
}

\author{
Paola Cúneo ${ }^{1}$ \\ CONICET / Universidad de Buenos Aires, Facultad de Filosofía y Letras, Instituto de Lingüística
}

Florencia Tola ${ }^{2}$

CONICET / Centro EREA del LESC, UPO/CNRS

Recibido: 25 de mayo de 2018; Aprobado: 8 de noviembre de 2018

Cómo citar este artículo / Citation: Cúneo, Paola y Florencia Tola. 2020. «Juego verbal, metáfora y sexualidad. El discurso de humor (nayakyaGak) entre los tobas (qom) del Gran Chaco sudamericano». Disparidades. Revista de Antropología 75(1): e005. doi: <https:// doi.org/10.3989/dra.2020.005>.

RESUMEN: El propósito de este artículo es analizar un tipo de discurso de humor (nayakyaGak) que forma parte del repertorio verbal de los tobas del Chaco argentino: las bromas sexuales. Integrando perspectivas lingüísticas y etnográficas, nos centramos tanto en los recursos léxicos y morfosintácticos utilizados en este tipo de nayakyaGak así como en sus contextos de interacción, con especial interés en las claves de contextualización que permiten construir el marco de juego de las bromas. Profundizaremos en las construcciones metafóricas que se encuentran en uso en las bromas sexuales y mostraremos posibles vínculos entre el campo semántico de la marisca (cacería) y el de la sexualidad. Así también, daremos cuenta de que el humor presente en las bromas que transcribiremos conforma una práctica discursiva que permite hablar de la sexualidad y la afectividad. El trabajo constituye un aporte al estudio del repertorio discursivo toba, así como también a las reflexiones antropológicas en torno a la sexualidad.

PALABRAS CLAVE: Humor; Sexualidad; Marco de juego; Toba; Guaycurú; Lenguas amerindias.

ABSTRACT: The purpose of this article is to analyse a type of humorous discourse (nayakyaGak) that is part of the Toba (Guaycuruan, Gran Chaco region, Argentina) verbal repertoire, namely sexual jokes. Integrating linguistic and ethnographic perspectives

\footnotetext{
Esta investigación se realizó gracias al apoyo económico del CONICET (Consejo Nacional de Investigaciones científicas y Técnicas de Argentina) y de la Universidad de Buenos Aires (Proyecto UBACyT 2016-2019 20020160100106BA)

1 Correo electrónico: pcuneo@gmail.com. ORCID iD: <https://orcid.org/0000-0001-7901-412X>.

2 Correo electrónico: tolatoba2015@gmail.com. ORCID iD: <https://orcid.org/0000-0002-6777-4575>.
} 
of analysis, we focus on the lexical and morphosyntactic resources used in this type of nayakyaGak as well as on its social functions and contexts of interaction, with a special focus on key contextualization factors creating a play frame for the jokes. Our analysis examines the metaphorical constructions behind sexual jokes and shows potential links between the semantic fields of subsistence practices and sexuality. Furthermore, we show that humour constitutes a discourse practice in which sexuality and emotion can be expressed. This paper contributes both to the study of the Toba discourse repertoire and to the anthropological discussion on sexuality.

KEYWORDS: Humour; Sexuality; Play frame; Toba; Guaycuruan; Amerindian languages.

Copyright: (C) 2020 CSIC. Este es un artículo de acceso abierto distribuido bajo los términos de la licencia de uso y distribución Creative Commons Reconocimiento 4.0 Internacional (CC BY 4.0).

Puns and jokes, like all speech play more generally, should not be viewed as marginal or insignificant communicative behaviour. These seemingly small and unimportant forms involve a condensation of basic processes and principles of discourse -such as cohesion, disjunction, frame construction and braking, and the relationship between the said and the unsaid- as well as psychological, interactional, and sociocultural underpinnings of discourse (Sherzer 2002: 52).

\section{INTRODUCCIÓN}

El propósito de este artículo es adentrarnos en un tipo de discurso de humor (nayakyaGak) que forma parte del repertorio verbal de los tobas (qom) del Chaco argentino -las bromas sexuales-, integrando las perspectivas de análisis lingüístico y etnográfico ${ }^{3}$.

El trabajo fue llevado a cabo principalmente con tres colaboradores tobas ${ }^{4}$ con quienes tenemos una rela-

3 Una versión previa -inédita- de este trabajo fue presentada en el Coloquio "Arte verbal y lenguas indígenas: aspectos metodológicos, tipológicos y de contacto", en el marco del XIV Congreso de la Sociedad Argentina de Lingüística (SAL XIV), Catamarca, 22 al 25 de abril de 2014. Agradecemos los comentarios y aportes de los participantes del Coloquio. Las dimensiones del humor sexual, las relaciones amorosas no oficiales y las prácticas chamánicas son abordadas en un trabajo más amplio desde una perspectiva predominantemente antropológica en Tola y Cúneo (2014).

4 Un joven maestro bilingüe y líder político de la Comunidad Riacho de Oro (Formosa) (VS), un anciano maestro de len- ción de largo plazo y de cercana intimidad. Con ellos, el tema de las bromas que los hombres se dicen entre sí surgió espontáneamente (trabajando temas relacionados con el erotismo y las relaciones) y luego fue específicamente trabajado en entrevistas abiertas posteriores. Si bien reconocemos que tanto en antropología como en lingüística el trabajo de investigación suele llevarse a cabo con un mayor número de interlocutores, en nuestro caso, la temática que decidimos abordar constituye en sí misma una limitación. Por un lado, no son muchos los hombres tobas que se atreverían a expresarnos bromas que, para ellos mismos, son de alto contenido sexual. Por otro lado, las bromas a las que accedimos no suelen ser expresadas ante mujeres. Es decir, la temática en la que decidimos adentrarnos no es de fácil acceso al ser nosotras investigadoras mujeres. Asimismo, si bien las mujeres tobas refieren ellas también bromas, nunca hemos presenciado bromas sexuales femeninas y, menos aún, bromas que involucren el mundo de la caza ya que dicha actividad es principalmente masculina. A pesar de estas limitaciones metodológicas, desde hace veinte años llevamos adelante nuestra experiencia etnográfica y etnolingüística entre los tobas. La continuidad y el tiempo prolongado de nuestras etnografías nos permiten entonces situar el tema acotado que trabajamos en este texto en relación con una gran variedad de ámbitos de la vida social, de las relaciones entre los géneros, de la sexualidad y la afectividad a las que accedemos en nuestros trabajos de campo.

El Gran Chaco sudamericano constituye una vasta llanura semiárida que se extiende sobre parte del norte de Argentina, del este de Bolivia, del oeste de Paraguay y del sur de Brasil, a lo largo de un millón de kilómetros cuadrados. Esta región se halla delimitada por los ríos Paraná y Paraguay hacia el este, por la pre-cordillera de los Andes hacia el oeste, por los llanos de Chiquitos y la meseta del Mato Grosso hacia el norte y por la cuenca del río Salado hacia el sur, representando una transición hacia la Pampa argentina. La lengua toba pertenece a la familia lingüística guaycurú (junto con el pilagá, el mocoví y el caduveo y las ya extintas abipón y mbayá). Los tobas son aproximadamente 127.000 personas (INDEC 2010) que habitan en el Gran Chaco (provincias argentinas de Chaco, Formosa y Salta), en el sudeste de Bolivia y en

gua qom oriundo de El Espinillo (noroeste de la provincia del Chaco) (MM) y un anciano experimentado en la caza y la pesca nacido en Pampa del Indio (centro-norte de la provincia del Chaco) (RY). 
las cercanías de El Cerrito (Paraguay). Como consecuencia de procesos migratorios, viven también en asentamientos próximos a las grandes ciudades. En estos contextos, la lengua indígena está siendo desplazada por el español en ciertos ámbitos comunicativos. No obstante, a pesar del largo proceso de contacto con la lengua mayoritaria, el repertorio verbal qom se manifiesta a través de variados estilos y géneros del habla -por ejemplo, el consejo (nqataGak), la rogativa (natamnaGak), la narración mítica (togeshec na?aqtagek) o la crónica histórica (dalaGayk IPaqtaGanaGak) - que son parte esencial de la vida social entre los qom. A través de la ejecución de estos géneros discursivos es que se transmiten y reproducen valores y conductas sociales (cf. Messineo 2014, para un acercamiento al arte verbal qom). En ellos, el humor (nayakyaGak), que constituye el foco de estudio de este trabajo, representa un factor de continuidad de las prácticas verbales y culturales qom.

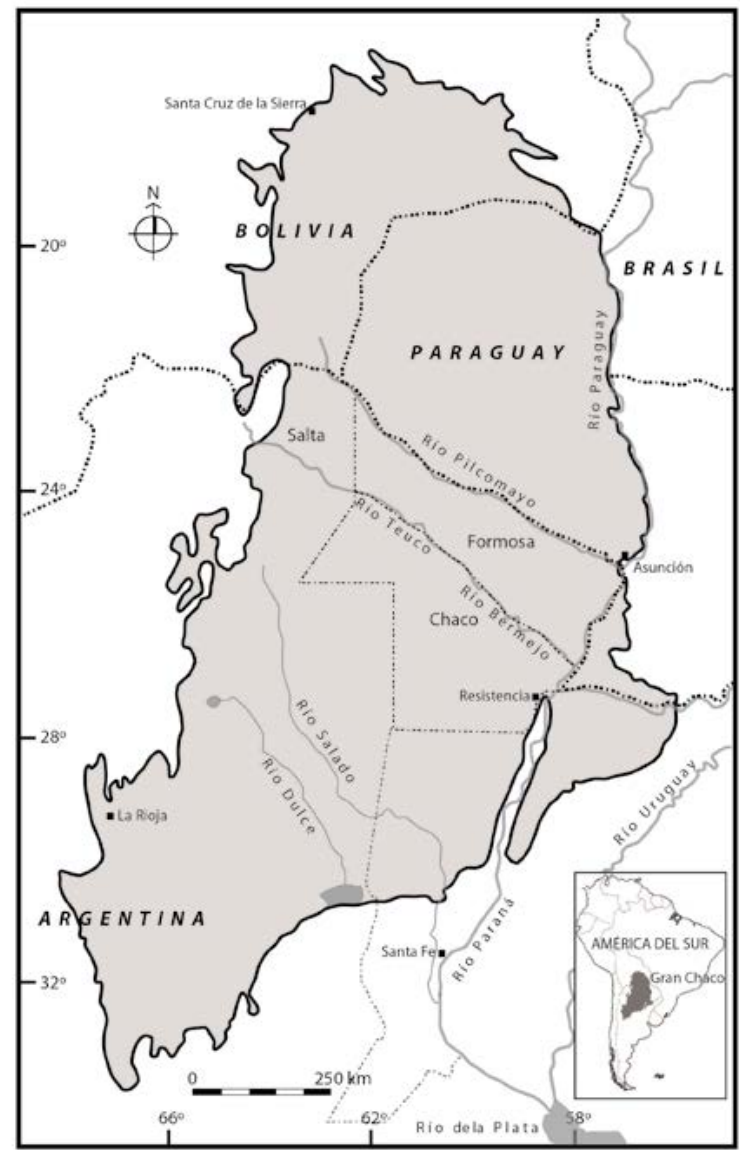

MAPA 1.- El Gran Chaco. Confección: Celeste Medrano.

En este trabajo analizaremos las bromas que los hombres se dicen entre sí, en ausencia de mujeres, que están elaboradas principalmente a partir de un lenguaje metafórico, si bien contextualmente ancladas mediante recursos deícticos. Las prácticas de subsistencia o marisca (caza, pesca, recolección y meleo) se constituyen como el dominio que permite a los hombres hablar, siempre de manera indirecta, de la sexualidad y del cuerpo femenino. Estas bromas puramente alusivas no refieren al ámbito del matrimonio sino al de las relaciones amorosas no oficiales, tanto prematrimoniales como extramatrimoniales. Nos proponemos mostrar que el humor presente en las bromas que analizaremos conforma una práctica discursiva que permite hablar de la sexualidad y la afectividad, y que se constituyen como formas tobas de conocimientoacción sobre los seres y las relaciones.

Varios estudios antropológicos han mostrado que abordar las formas características del humor de una sociedad puede permitir una mejor comprensión de la misma. En antropología, son pioneros los trabajos de Radcliff-Brown (1940, 1949) y Mauss (1926) así como los de diversos africanistas (Paulme 1939; Moreau 1941, 1944, entre otros) sobre las joking relationships. Dentro de las investigaciones realizadas en sociedades indígenas de las tierras bajas sudamericanas, se destacan los trabajos de Overing y su escuela (2000) sobre una «verdadera epistemología del ridículo» (Lagrou 2006: 60) ${ }^{5}$. Overing muestra la pertinencia epistemológica de indagar el «porqué los mitos piaroa enfatizan lo risible, lo erótico y lo obseno» (ibid.). Existe, tal como sostiene Lagrou, una «filosofía política por detrás de estas estéticas de lo grotesco» (ibid.). En un trabajo reciente, Emmanuel de Vienne retoma las joking relationships entre los trumai de la Amazonía brasileña del Alto Xingú, aporta a la definición de un modelo del humor y del marco de juego, y al mismo tiempo delimita relaciones culturalmente específicas. Para la comprensión de estos fenómenos, las distintas investigaciones insisten en la necesidad y el valor de descripciones etnográficas detalladas sobre el humor y la broma, tal como señalan Carty y Musharbash (2008: 209).

Desde la perspectiva de la antropología lingüística, el ya clásico trabajo de Keith Basso (1979) discute los aspectos lingüísticos y comunicativos de las bromas entre los apache occidentales (Western Apache o

5 Cabe mencionar las interpretaciones de Lévi-Strauss en las Mitológicas que vinculan la risa con los orificios del cuerpo (1964) y el trabajo reciente de Rosengren sobre la risa entre los matsiguenga (2010). 
ndee) acerca de los 'blancos'. Para su análisis, el autor pone de relieve el conocimiento presupuesto y compartido por los hablantes, que toma la forma de un "texto secundario», imprescindible para ejecutar e interpretar una broma. La categoría analítica de texto secundario refiere al conocimiento compartido $y$ a las presuposiciones que deben existir entre los interlocutores (cf. Bateson 1972; Basso 1979; Black 2012) ${ }^{6}$. Cuando se ejecuta una broma, la audiencia puede creer que la ejecución es seria. No obstante, el aspecto humorístico de una broma depende del reconocimiento de la audiencia de que existe un conocimiento compartido necesario para decodificar presuposiciones y reconocer la relación entre el texto secundario y el texto primario (Bateson 1952 en Black 2012: 89). De esta manera, la broma está íntimamente ligada a la presuposición; a lo no dicho. Ahora bien, el texto secundario solo se interpreta como tal dentro de un marco de juego [play frame] -concepto que utilizaremos en el análisis de las bromas qom-.

El concepto de marco o frame -introducido por Erving Goffman (1974) desde la sociología- provee una guía para interpretar la acción y la interacción social. Un discurso puede estar anclado a un marco específico a través de diferentes recursos o claves, de manera tal que los participantes son dirigidos a interpretar las acciones humanas de una manera u otra. Las claves o recursos que pueden permitir enmarcar un discurso son, por ejemplo, una afirmación convencionalizada, un gesto, una expresión facial, la orientación corporal, la prosodia o incluso la secuencia de turnos de habla que pueden marcar un momento en el que la interacción cambia a un marco de juego o performance (Goffman 1974: 44). Desde el enfoque de la antropología lingüística, Joel Sherzer define el marco [frame] como «la definición, concepción u organización de una actividad, ya sea real o literal, ensayada, practicada, comentada, mentida, soñada o fantaseada. El juego es entonces un tipo de marco» (2002: 14; traducción nuestra).

En línea con un extenso recorrido en la literatura sobre humor centrada en la noción de «incongruen-

6 En términos de Basso, «any actual performance may be said to consist in the construction and presentation of a secondary text that is intended to be understood as a facsimile or transcript copy of the primary text on which it is patterned. [...] when secondary texts are read as primary ones and the joking frame 'breaks', the consequences can be explosive» (1979: 41-43). cia» [incongruity] como modelo para caracterizar una broma (cf. Morreall 1987, 1989; Attardo 1997), WiIliam Beeman explica el funcionamiento del humor en términos comunicativos a partir de la idea del desplazamiento de marcos de referencia:

Un actor comunicativo presenta un mensaje $u$ otro material de contenido y lo contextualiza dentro de un 'marco' cognitivo. [...] Luego, él o ella, de repente, deja de lado este marco, y revela uno o más marcos cognitivos adicionales que se muestran a los miembros de la audiencia como posibles contextualizaciones o reformulaciones del contenido original. La tensión entre el encuadre [framing] original y el repentino reencuandre [reframing] da como resultado una liberación emocional reconocible como una respuesta de disfrute que se traduce en sonrisas, risas y gozo (Beeman 2000: 103, traducción nuestra).

Esta tensión basada en un double-framing de una palabra o de una expresión es entonces la fuerza motora que subyace al humor. La yuxtaposición de marcos interpretativos es lo que produce el humor ${ }^{7}$.

En esta misma línea, el trabajo reciente de Steven Black (2012) integra los modelos teóricos sobre juego, ejecución y humor para examinar las propiedades lingüísticas y comunicativas de las bromas que ejecutan los zulu-hablantes sudafricanos en un coro evangélico para portadores de VIH. Su objetivo es teorizar acerca de por qué la broma -y de manera general, el humorse utiliza para referirse a temas que están en las fronteras de lo que no se considera aceptable hablar en otros contextos. Nos interesa el estudio de Black dado que sugiere que las propiedades discursivas analizadas entre los zulu-hablantes pueden actuar, en otras culturas, en diversos géneros humorísticos, en los cuales los individuos se aproximan a temas que son embarazosos, incómodos, tristes o tabú.

Finalmente, destacamos que el humor ha sido definido como uno de los actos comunicativos que depende más fuertemente de la participación cooperativa del ejecutante y la audiencia (Beeman 2000). Para

7 Según Beeman (2000), el humor presenta típicamente cuatro etapas: el setup, la paradoja, el desenlace [dénouement] y la descarga [release]. Por su parte, Sherzer (1985) define las bromas como unidades discursivas que consisten en dos partes, el set y el remate [punchline]. Como veremos, estas etapas no se presentan claramente en la ejecución del tipo de bromas qom que analizamos. Aclaramos que las traducciones de Beeman a lo largo del texto corresponden a las autoras. 
poder disfrutar del humor, la audiencia debe captar el chiste. El ejecutante, por su parte, para que la broma funcione debe tener en cuenta -como mencionamos- el conocimiento compartido con la audiencia.

Dentro de los estudios antropológicos y lingüísticos chaqueños, el humor no se constituyó como un tema de análisis. Desde una aproximación al arte verbal, existen referencias a relatos humorísticos (especialmente, los relatos del zorro travieso, cf. Wilbert y Simoneau 1992; Gordillo 2005; Messineo 2014, entre otros) pero no se profundiza el análisis sobre el humor en sí. Desde la antropología hay algunas referencias aisladas al humor chorote y nivaclé en relación al erotismo (especialmente el concepto de «erotismo lúdico» de Siffredi y Cordeu 1992) y a las relaciones de plaissanterie en la vida cotidiana de los ayoreo (Otaegui 2014, 2016). No obstante, estos estudios no abordan aspectos lingüísticos sobre el humor. Asimismo, es de destacar la compilación de narrativa erótica chorote y nivaclé realizada por Chase-Sardi, Siffredi y Cordeu (1992). Estos autores al igual que Idoyaga Molina (1976), De los Ríos (1978-1979) y Braunstein (1983) reconocieron la existencia de «celebraciones rituales fuertemente orgiásticas; y también, el reconocimiento institucionalizado de ciertos tipos de posesión erótica» (Siffredi y Cordeu 1992: 18).

Entre los qom el erotismo se asocia con diversas instituciones sociales (el parentesco, la afectividad, la salud, la caza, la guerra, entre otros) que ponen en juego sentidos semejantes. Del respeto de las prohibiciones sexuales depende, por ejemplo, la salud y la vida de la mujer, del esposo, del bebé lactante y del recién nacido. El respeto de las prohibiciones sexuales se asocia también con la afectividad ya que una madre que, desde la perspectiva toba, quiere a sus hijos no mantendrá relaciones sexuales durante la lactancia a los fines de evitar el nacimiento de otro hijo y el destete del lactante. Tal como expresan Siffredi y Cordeu, "el lenguaje y símbolos de esas [otras] manifestaciones [sociales] se trasvasan de continuo de una a otra y sus significados se realimentan recíprocamente» con el del erotismo (1992: 13-14). Asimismo, la sexualidad y el género no son solo centrales en la vida de los humanos y en las relaciones matrimoniales o extramatrimoniales sino que atraviesan la vida social de los no-humanos.

Abordaremos a continuación algunas de las bromas sexuales y mostraremos cómo opera para la ejecución e interpretación de las mismas el conocimiento presupuesto y compartido relativo a la cacería y a la comida.

\section{BROMAS SEXUALES QOM. ENTRE EL LENGUAJE FIGURADO Y EL ANCLAJE DEÍCTICO}

Las bromas sexuales que los hombres se dicen entre sí constituyen un tipo de discurso de humor o nayakyaGak ${ }^{8}$ que forma parte del repertorio verbal toba. El término nayakyaGak deriva del verbo dayaki que significa '(él) juega, bromea, hace un chiste'. Con respecto al contexto de interacción, las bromas sexuales que analizamos requieren de una relación simétrica entre un ejecutante o bromista (nayakyaGayk) y un receptor o audiencia. Típicamente, dicha relación es de compañerismo y complicidad, y ambos interlocutores son masculinos. Además, es propio de la situación comunicativa de las bromas que la mujer de la cual se habla circule cerca del bromista, a la vista pero a una cierta distancia como para que no alcance a oír.

Veamos un ejemplo de la situación de broma entre dos compañeros que se encuentran en un lugar público:

(1)

$\begin{array}{lll}\text {-La? } & i \text {-zik } & \\ \text { INTERJ } & \text { 1POS-amigo } & \\ \text { 'Hola, amigo.' } & & \\ \text {-Nege? } & k a & \text { Pa-lotayke } \\ \text { INTERR } & \text { D:aus } & \text { 2A-buscar } \\ \text { '¿Qué estás buscando?' } & \end{array}$

¿¿ué estás buscando?'

$\begin{array}{lll}\text {-Ñi-Pep-tak... } & \text { se-lotayke } & \text { ñilimshi-lyale } \\ \text { 1M-cazar-PROG } & \text { 1A-buscar } & \text { corzuela-DIM.F }\end{array}$

'Estoy cazando... estoy buscando una corzuelita [Mazama americana].'

(Fig. 'Estoy intentando conquistar... estoy buscando una chica')

Un aspecto que define este tipo de bromas es que ellas son pronunciadas con un tono serio. Quien las ejecuta no se ríe al decirlas, sino que habla con seriedad, siendo posible que quien las escucha no entienda el sentido figurado que contienen. En palabras de uno de nuestros interlocutores: «está hablando de otra cosa». De hecho, es necesario (re)conocer la situación, el género y el marco comunicativo para comprender el sentido sexual de estos comentarios que, fuera de un contexto jocoso y tácito, dejan de

8 Asimismo, los relatos del zorro travieso -documentados en Messineo (2014)- constituyen otro tipo de discurso humorístico que puede incluirse también dentro del género nayakyaGak. 
ser bromas para volverse expresiones que remiten a otras situaciones de la vida social.

Ahora bien, ¿cuáles son estas situaciones? Casi todas ellas pertenecen al ámbito de las actividades de obtención de alimento. De hecho, este tipo de bromas se sirve del lenguaje de la marisca, dominio ampliamente conocido por los hombres, para aludir a las prácticas sexuales y al cuerpo. Como mostraremos, en las bromas los hombres son quienes aciertan los tiros de sus armas de fuego, tiran la red, pescan con lanza o anzuelo y extraen la miel contenida en las bolsas con las que la juntan. Son sus compañeras sexuales -aunque no nombradas más que por omisión y partes del cuerpo- el objeto de estas acciones masculinas.

Describiremos ahora dos aspectos pragmático-discursivos que permiten caracterizar sólidamente estas bromas: por un lado, la construcción exclusivamente figurada de este discurso erótico y, por otro, su anclaje deíctico a la situación comunicativa.

\section{METÁFORA}

Desde la perspectiva de Lakoff y Johnson (1980), la metáfora no es una cuestión exclusivamente de lenguaje sino del pensamiento y la acción, y su estudio permite acceder al sistema de valores del grupo que las gesta y utiliza. De hecho, desde este enfoque, no existen semejanzas dadas entre las cosas, sino que las mismas son construidas de manera sistemática en cada lengua y en cada cultura.

En términos generales, las metáforas se basan en información de un dominio conocido para estructurar una aserción sobre propiedades de otro dominio que en principio resulta más difícil de conceptualizar. Este proceso de extensión metafórica consiste en proyectar características de un dominio fuente hacia un dominio meta. Los dominios fuente son típicamente experiencias cotidianas, generalmente aquellas del mundo físico. Mediante este mapeo de ciertas propiedades salientes de un dominio fuente hacia un dominio meta, la metáfora permite entender ciertos fenómenos ${ }^{9}$.

9 Las metáforas constituyen entonces formas de usar el lenguaje en contextos situacionales y culturales específicos (Mühlhäusler 2012: 3). Por ejemplo, en el trabajo de Sherzer (1992 [1983]) sobre las formas del habla kuna en Panamá, el autor muestra el uso de lenguaje simbólico figurado en el discurso político. Este discurso se conforma en
Veamos en detalle algunas expresiones tobas:

(2)

$\begin{array}{lll}\text { Negert } & k a & \text { Par-apayigi-shek } \\ \text { INTERR } & \text { D:aus } & \text { 2POS-hincar-NMz }\end{array}$

‘¿ué es tu hincado?' (Fig.: '¿qué es tu hijo?')

De este modo chistoso, un hombre le está preguntando a otro sobre el sexo de su hijo ('¿qué es tu hijo, varón o mujer?’). Observemos que la expresión nominal Para?ayigishek 'hincado' remite al resultado de la acción del verbo 'hincar' (según la RAE, «introducir o clavar algo en otra cosa»). En este caso, la pregunta por el sexo del hijo se formula aludiendo al acto sexual con la madre del mismo, acto sexual sugerido a partir del verbo 'hincar' nominalizado. Por lo tanto, el hijo es nombrado como la consecuencia de haber 'hincado' a una mujer. En esta pregunta, ni la madre ni el hijo ni el acto sexual son nombrados directamente. Ellos son aludidos a partir de una expresión que proviene del ámbito de la pesca. Los peces se 'hincan' con la lanza de pesca o fija, y cuando un pescador extrae la fija del agua, otro pescador suele preguntarle nege?t ka ?araßayigishek? ('¿qué es tu hincado?') para saber qué clase de pez extrajo del agua.

El mundo de la pesca que se lleva a cabo con lanza (latakyaGay: su fija o lanza), red (lalegek: su red) o anzuelo (lqoyna: su anzuelo) sirve también para aludir a la acción de buscar una compañera sexual. Cuando un hombre camina solo por la comunidad, sin rumbo, puede venir otro hombre y preguntarle:

(3)

Paw-aleg-aGa-tak ke?e
2A-red-VBZ-PROG INTERR
'¿Estás tirando la red [redeando]?'
(Fig.: '¿Estás buscando compañera?')

Esta pregunta y su respuesta pueden formularse también a partir del verbo 'pescar con anzuelo', tal como se aprecia en la siguiente respuesta:

(4)

$\begin{array}{ll}\text { ayem } & \text { se-qoyna-Ga-tak } \\ \text { PRON1 } & \text { 1A-anzuelo-VBZ-PROG }\end{array}$

'Yo estoy pescando (con anzuelo) [anzueleando].'

(Fig.: 'Estoy buscando compañera')

kuna mediante metáforas y otras figuras sobre los jefes y otros líderes políticos, mayormente extraídas del mundo vegetal y animal kuna, para representar la estructura política kuna. 
El mundo de la caza suele ser también usado para remitirse a las relaciones sexuales entre hombres y mujeres, más precisamente a la eyaculación:

(5)

$\begin{array}{llll}\text { a-so } & \text { t-ay-gi } & \text { so } & \text { y-aynaGan-aqte } \\ \text { F-D: dist } & \text { 3-ir-DIR } & \text { D:dist } & \text { 1Pos-disparar-INS } \\ \text { 'En esa [mujer] entraron mis tiros/disparos.' } \\ \text { (Fig.: 'En esa [mujer] }\end{array}$

Los tiros (yaynaGanaqte) remiten, según el contexto, a los proyectiles de las armas de fuego, a los tiros de flecha o a los disparos de la 'gomera', así como a la eyaculación durante el acto sexual. Para aludir a la relación sexual sin hablar directamente de ella, se equiparan los proyectiles del arma de fuego tirados a una presa con la eyaculación. La acción de acertarle con los tiros a una presa es usada indirectamente para referirse al acto sexual o al hecho de acertarle a una mujer con los 'tiros de semen':

(6)

$\begin{array}{llll}\text { ayem } & \tilde{n} \text {-aten } & \text { a-so } & \text { qa?añole } \\ \text { PRON1 } & \text { 1M-acertar (a una presa) } & \text { F-D:dist } & \text { joven } \\ \text { 'Yo le acerté a esa joven.' } & & \end{array}$

Si dos hombres comentan acerca de otro que mantiene relaciones con una mujer mayor que él, el comentario chistoso que son susceptibles de hacer es el siguiente:

$\begin{array}{lll}\text { n-amarek-tak } \quad \text { a-so } & \text { I-kote } \\ \text { 3M-raspar-PRoG } & \text { F-D:dist } & \text { 3Pos-abuela } \\ \text { 'Está raspando a la abuela.' } & \end{array}$

La expresión 'raspar una lota' (namarektak añi lota) ${ }^{10}$ remite a la acción destinada a extraer lo máximo posible de la miel que queda contenida entre los pelos del cuero de animal. Al raspar la bolsa, los cazadores se esfuerzan por obtener lo máximo de miel, de algo que es considerado, junto con la algarroba (amap), lo dulce ( $a m a$ ?) por excelencia. La expresión usada para referirse a la relación sexual con una anciana se relaciona con el trabajo de raspar al extremo la bolsa para obtener algo de miel, en sentido figurado, de placer.

En este mismo ámbito de significados, uno de los términos que se usa para describir el placer sexual proviene del ámbito de la comida. Ama? ('dulce', 'rico') se emplea para expresar el placer que produce un alimento rico -o muy dulce- así como el placer que se

10 La lota es la bolsa hecha con cuero de animales que los qom usan para cargar la miel que sacan en el monte. siente durante una relación sexual. ?am $a m a^{\prime}$ (lit. 'vos sos dulce/rica') puede ser una frase expresada durante el encuentro sexual. Existen otras expresiones que provienen del mundo de la alimentación y que se usan en contextos sexuales. Por ejemplo:

(8)

$\begin{array}{ll}\text { zi-shetayke } & \text { l-apat } \\ \text { 1I-desear/querer } & \text { 3Pos-carne } \\ \text { 'Quiero [comer] carne.' } & \\ \text { (Fig. 'Quiero tener relaciones sexuales') }\end{array}$

(9)

$\begin{array}{lll}\tilde{n} \text {-aman } & k a & \text { l-apat } \\ \text { 1M-gustar } & \text { D:aus } & \text { 3Pos-carne } \\ \text { 'Me gusta la carne.' } & \end{array}$

(Fig. 'Me gusta tener relaciones sexuales')

Como vemos, la asociación entre sexualidad y alimentación (ampliamente referida para otras regiones, cf. Gregor 1985; Gregor y Tuzin 2001) cobra particular relevancia entre los qom y se lee tanto en las bromas como en las expresiones referidas. Esta asociación se constata también en prácticas cotidianas, por ejemplo, en las prohibiciones alimenticias que existen en etapas específicas del ciclo reproductivo femenino; momentos en los que también rigen las prohibiciones sexuales. Durante el embarazo cuando el hombre y la mujer nutren gradualmente al nuevo ser a partir de sus fluidos sexuales ${ }^{11}$ - la mujer debe abstenerse de comer carne de animales silvestres y pescado ${ }^{12}$ bajo riesgo de causar malformaciones físicas o trastornos comportamentales en el bebé a raíz de la posible fusión entre el niño y el espíritu del animal ingerido (proceso conocido como nawoga, 'contagio' o 'influencia') ${ }^{13}$. El hombre, por el contrario, debe procurar ingerir pescado ya que se sostiene que el aumento en la producción de semen está íntimamente ligado al consumo de este alimento. La mujer debe evitar acercarse a todo curso de agua,

11 Cabe destacar que, para los qom, un solo acto sexual no basta para crear un bebé. La constancia de las relaciones sexuales por un período de tiempo variable es necesaria para comenzar a formar un nuevo ser. Estas ideas son comunes a una variedad de sociedades indígenas (cf. Hugh-Jones 1979; Viveiros de Castro 1992; Gregor 1985; Jolly 2001).

12 Entre los mehinaku, tal como refiere Gregor, pescar es la actividad masculina por excelencia y el pescado es el «símbolo primario de la sexualidad masculina» (1985: 79, traducción nuestra).

13 Sobre este proceso, diversos especialistas de la región han brindado detalles e interpretaciones. Cf. Balducci (1982); Tola (2004); Wright (2008); Medrano (2013), entre otros. 
así como tocar las armas de caza y pesca del marido durante el embarazo y la menstruación bajo riesgo de causar enfermedades en su esposo, mala suerte en la caza y catástrofes para su comunidad.

Si la asociación entre el hombre y el pescado se establece a partir de la reproducción, la concomitante disyunción entre la mujer, el agua y el pescado se produce por la asociación entre la sexualidad, la alimentación y la salud-enfermedad. Sin duda, la sexualidad femenina -especialmente sus fluidos- es considerada por los hombres como fuente de peligro y contaminación, hecho que se observa en las restricciones y reclusiones a las que las mujeres deben someterse. El control femenino de su sexualidad y del correcto manejo de los fluidos corporales es percibido también como fuente de vida y salud. De hecho, el respeto de las prohibiciones sexuales por parte de las mujeres es lo que garantiza la vida, el buen crecimiento y la salud de los hijos. En el respeto de las mismas se traslucen, como mencionamos, aspectos de la afectividad ya que una madre preocupada por la vida y la salud de sus hijos es aquella que cumple con las restricciones alimenticias y sexuales, no vistas tanto como formas contraceptivas sino como instancias que generan human and cosmic fecundity (Jolly 2001: 178) ${ }^{14}$.

La asociación entre sexualidad, cacería y alimento se observa no solo en la selección lingüística utilizada en las bromas sino también en las prácticas y prescripciones sociales que hemos puesto de relieve. En efecto, según Lakoff y Johnson (1980), los procesos de metáfora y metonimia tienen sus raíces en la experiencia tanto física como social y cultural, y la selección lingüística presente en las metáforas evidencia qué aspecto es central para una determinada sociedad. Los ejemplos brindados expresan que la elección de las metáforas que vinculan dominios sexuales y alimenticios no es aleatoria, sino que refleja formas qom de entendimiento.

El mismo proceso metafórico se observa cuando, en las bromas y expresiones cotidianas, se mencionan las partes del cuerpo sexuado:

14 Cabe mencionar que la restricción sexual durante toda la lactancia está destinada a evitar un nuevo embarazo que ocurriría por mantener constantes relaciones sexuales. El nuevo embarazo afectaría la salud del bebé lactante a raíz de los ataques del espíritu del bebé en gestación hacia él. Estos ataques -que suelen ocurrir en sueños- se expresan también a través de la leche materna que deja de nutrir al bebé por tornarse "acuosa y transparente», según expresan las mujeres tobas.
(10)

damaze woro so l-yoG-osoGon-aGa-ki

PRON3 EX D:dist 3POS-cargar-CAUS-NMZ-LOC

'Ese tiene su lugar de carga' (Fig.: 'vagina/útero')

Mediante esta expresión los hombres refieren a que otro hombre ya consiguió una compañera sexual. Recordemos que la expresión en español regional para referirse al útero es 'bolsa' o 'lugar de carga' (lyoGosoGonaGaki) y que dicho vocablo puede estar vinculado a la idea de que la gestación se produce tras numerosos actos sexuales en los que el semen se deposita en una especie de bolsa hasta comenzar a dar forma al bebé junto con la sangre menstrual.

$\begin{array}{lc}\text { alo } & \text { l-o?o } \\ \text { mujer } & \text { 3POS-cuidador/especialista } \\ \text { 'pene' (Lit. 'especialista en mujer') }\end{array}$

El término lo?o posee el significado básico de 'cuidador' o 'encargado', aunque algunos hablantes lo traducen como 'especialista', en particular para referirse a una actividad de caza, meleo o recolección (por ej.: mañik lo?o 'especialista en ñandú' o amap lo?o 'especialista en algarrobo'). También puede ser usado con el significado de 'arma' (por ejemplo, sa ishet da lo?o 'no tenía arma'). En síntesis, este término posee una fuerte asociación con los dominios de la marisca, la predación y la alimentación (cf. Cúneo 2013) ${ }^{15}$.

En síntesis, estas formas lúdicas de interacción verbal, al ser contextualizadas etnográficamente, revelan y destacan aspectos importantes de la vida social y cultural entre los qom. A su vez, observamos que estas bromas poseen la función social de mantener y reforzar la asociación entre marisca y sexualidad ${ }^{16}$.

\section{DEIXIS}

A pesar de que -como observamos- las bromas se ejecutan en base a un lenguaje tácito, no explícito, y aunque pueda parecer contradictorio, este tipo de discurso posee un fuerte anclaje deíctico a la situa-

15 Ver específicamente Cúneo (2013: 274-277) para el uso de este término en el vocabulario etnozoológico, en el que también se codifican estas relaciones.

16 En cuanto a la relación con la masculinidad, no fue nuestro interés delimitar el ámbito de lo masculino/femenino, y entendemos que es necesario un trabajo etnográfico en profundidad sobre este aspecto para el caso de los grupos qom/toba con los que trabajamos. 
ción de habla. Se entiende por deixis toda expresión lingüística que en una lengua localiza en el espacio y en el tiempo las circunstancias del evento de habla y a sus participantes (hablante, oyente y referente). Se pueden distinguir tres grandes categorías de deixis: la deixis personal, la espacial y la temporal (Anderson y Keenan 1985).

El toba, como el resto de las lenguas guaycurúes, presenta un sistema de seis clasificadores deícticos o demostrativos (cf. Tabla 1) que se anteponen a los nombres y que codifican rasgos configuracionales y deícticos. Indican, por un lado, las distancias espaciales y/o temporales del referente con respecto al hablante (próximo, distante y ausente) y, por otro, características relacionadas con la forma o la posición del objeto al que se hace referencia (vertical o parado, horizontal o acostado y tridimensional o sentado) (cf. Messineo 2003) ${ }^{17}$.

\begin{tabular}{|l|c|c|}
\hline & masculino & femenino \\
\hline $\begin{array}{l}\text { próximo, en movimiento } \\
\text { (acercándose al hablante) }\end{array}$ & na & ana \\
\hline $\begin{array}{l}\text { distante, en movimiento } \\
\text { (alejándose del hablante) }\end{array}$ & so & aso \\
\hline fuera de la vista, ausente & ka & aka \\
\hline parado [extendido vertical] & da & ada \\
\hline $\begin{array}{l}\text { acostado [extendido } \\
\text { horizontal] }\end{array}$ & zi & azi \\
\hline sentado [trideminsional] & ñi & añi \\
\hline
\end{tabular}

TABLA 1.- Clasificadores deícticos tobas.

En las bromas tobas aparece de manera predominante el uso de estos deícticos (y las formas derivadas) que, junto a los pronombres personales, permiten en primer lugar conectar la expresión lingüística con los participantes del evento de habla (deixis personal). Los pronombres de primera persona (ayem, en (13), por ejemplo), así como las formas pronominales de primera persona en los verbos refieren al hablante (masculino) mientras que los demostrativos (aso, asa?aso, en ejemplos (13) y (14)) designan al participante (femenino) del cual se habla. En estos últimos casos, aparece tam-

17 Los clasificadores deícticos funcionan como base para derivar los pronombres demostrativos; por ejemplo, ne?ena 'este (acercándose)', se?eso 'ese (alejándose)', de?eda 'ese (parado)', azi?azi 'esa (acostada)', etc.) así como el pronombre de 3 a persona (por ejemplo, namaze 'él (acercándose)', adamaze 'ella (parada)', añimaze 'ella (sentada)', zimaze 'él (acostado o muerto)', etc. bién codificado el género femenino que proporciona la información de género (deixis social). En la siguiente expresión, es claro que el demostrativo aka tiene un referente femenino:

$\begin{array}{llll}\text { ayem } & \tilde{n} \text { i-Pepe } & \text { ayem } & \text { s-ewota?a } \\ \text { PRON } & 1 \mathrm{M} \text {-cheknek } & \text { PRON } & \text { 1A-llevar POS1-arco }\end{array}$

'Voy a cazar llevo mi arco,'

$\begin{array}{llll}\text { sapeke?t } & \text { woro } & a-k a & \text { s-awaq-te-get } \\ \text { ojalá } & \text { EX } & \text { F-D:aus } & \text { 1A-encontrar?-DUR-DIR }\end{array}$

'ojalá que haya una con la que me cruce.'

Así también, los nombres de animales que refieren de manera figurada a la mujer son siempre femeninos: ñi?imshi-lyale 'corzuelita' (ejemplo (1)); qagetalyale 'ovejita' (ejemplo (18) infra). Como se observa, se evita la mención léxica explícita de hombres y mujeres particulares y del vínculo que los une. De hecho, la evitación verbal es la regla; la explicitación parece ser superflua. En algunos casos, las formas de referencia genérica como aso qa?añole 'esa joven' o bien las nominalizaciones ${ }^{18}$ metafóricas como lyoGosoGonaGaki 'su lugar de carga' permiten completar esta función de evitación verbal.

Veamos algunos ejemplos (rescribimos ejemplos (6) y (5) supra):

$\begin{array}{llll}\text { ayem } & \tilde{n} \text {-aten } & \text { a-so } & \text { a } a \text { añole } \\ \text { PRON1 } & \text { 1M-acertar (a una presa) } & \text { F-D:dist } & \text { joven }\end{array}$
'Yo le acerté a esa joven'.

$\begin{array}{llll}\text { asaPaso } & t \text {-ay-gi } & \text { so } & \text { y-aynaGan-aqte } \\ \text { DEM:dist.F } & \text { 3-ir-DIR } & \text { D:dist } & \text { 1POS-tirar-INS }\end{array}$

'En esa entraron mis tiros'.

Los demostrativos, como vimos, localizan a los participantes del evento de habla y así también ubican al evento narrado en el espacio (deixis espacial), dado que combinan la dimensión espacial con otras como el movimiento y la visibilidad. En varias de las bromas registradas (por ejemplo, en (13)) aparece el demostrativo femenino aso, que en esta lengua indica movi-

18 La nominalización es un recurso muy utilizado en las lenguas con diversas funciones discursivas. Entre ellas, las nominalizaciones que derivan de verbos permiten nombrar el evento o la acción (expresados en la base verbal) sin necesidad de mencionar a los participantes involucrados (quién hizo la acción, a quién, etc.); tanto el agente como el paciente pueden ser recuperados del contexto. 
miento y distancia con respecto al hablante. La deixis espacial permite definir la situación de habla que caracteriza a las bromas: la mujer sobre la que se habla circula alejándose de los interlocutores que bromean.

Finalmente, el aspecto verbal codificado en el verbo también expresa un tipo de deixis temporal. El morfema -tak denota un evento que posee una continuidad en el tiempo ${ }^{19}$. Entendemos que la continuidad de la acción se interpreta pragmáticamente como cercana al momento de habla. Por ejemplo:

$\begin{array}{ll}\text { ayem } & \text { se-qoyna-Ga-tak } \\ \text { PRON1 } & \text { 1A-anzuelo-VBz-PROG }\end{array}$

'Yo estoy pescando (con anzuelo).'

$\begin{array}{ll}\text { Paw-aleg-aGa-tak } & \text { ke?e } \\ \text { 2A-red-VB-PROG } & \text { INTERR } \\ \text { ‘¿Estás tirando la red?’ } & \end{array}$

$\begin{array}{lll}n \text {-amarek-tak } & \text { a-so } & \text { l-kote } \\ \text { 3M-raspar-PROG } & \text { F-DD } & \text { 3POS-abuela }\end{array}$

'Está raspando a la abuela.'

\section{EL MARCO DE LAS BROMAS}

¿Qué es lo que hace que se trate de una broma y no de una mera emisión de información sobre la práctica de la marisca? Y, ¿de qué manera se invoca un marco de juego particular que permite interpretar las emisiones como bromas y no como comentarios (literales, referenciales) sobre la marisca? Tomaremos el concepto de «marco» que introdujimos arriba para profundizar la definición de este tipo de bromas qom.

En el trabajo fundador de Bauman (2002 [1975]) sobre el arte verbal como ejecución, el autor pone en juego la idea de que la ejecución representa una transformación de los usos referenciales básicos del lenguaje; en palabras de Bauman: «hay algo que tiene lugar en el intercambio comunicativo que le dice al oyente: "interprete lo que digo en un sentido especial; no lo tome como queriendo decir lo que transmitirían las palabras solas, tomadas literalmente"» (2002 [1975]:

19 Este sufijo se aplica a verbos cuyo contenido léxico refiere a procesos dinámicos, a situaciones que pueden ser concebidas progresivamente o que implican el logro de un objetivo final (Messineo 2003: 76)
28). En este sentido es que «la ejecución establece un marco interpretativo dentro del que deben entenderse los mensajes comunicados, y este marco contrasta con, por lo menos, otro marco, el marco literal» (ibid.). Además, los marcos son metacomunicativos por definición, es decir que dan instrucciones al receptor y le son útiles para comprender los mensajes (Bateson 1972: 215-216 en Bauman 2002 [1975]: 33).

Ahora bien, ¿de qué manera se invocan, se establecen y se desplazan los marcos? ¿Qué recursos comunicativos constituyen claves para la ejecución? ${ }^{20}$ Asumimos que el encuadre está culturalmente convencionalizado, y cada comunidad de habla hace uso de un conjunto estructurado de medios comunicativos distintivos con el fin de dar la clave al marco (cf. Bauman 2002 [1975]) Entre los recursos formales y convencionales que pueden utilizarse se encuentran, por ejemplo, las fórmulas de apertura o cierre, la rima, la armonía vocálica, el paralelismo, ciertas pautas prosódicas especiales de tempo, acento, entonación, etc. (Bauman 2002 [1975]: 33). Con respecto al marco del humor, Jennifer Hay (1995: 10) -en un estudio sobre el uso del humor en grupos de amigos jóvenes hablantes de inglés en Nueva Zelanda- registra la selección léxica, el uso de eufemismos, la entonación, el uso de marcadores discursivos de informalidad, los gestos y expresiones cómicas, la exageración y la interacción con la audiencia, el estilo casual y familiar como características que permiten distinguir el discurso serio del humorístico.

Con respecto a las bromas sexuales qom -que los propios hablantes definen como parte del género nayakyaGak (del verbo dayaki 'juega')-, sugerimos que la ejecución es puesta en clave principalmente por el lenguaje figurado que analizamos: este resulta el recurso estilístico preferido y el medio comunicativo convencionalizado que permite activar el marco de la broma. De esta manera, la alusión e indirección funcionan como tipos de juego verbal que ponen en ejecución el marco de la broma. Además, tanto el uso del género femenino como de los clasificadores deícticos y otros demostrativos constituyen recursos gramaticales que funcionan también como claves de contextualización que son usados por los interactuantes para conformar el marco interpretativo. Es este marco de juego y broma el que deviene en un locus potencial para expresar aquello que de otra forma no es expresable.

20 En términos de Goffman (1974), ¿cómo la ejecución es puesta en clave [keyed]? 
No obstante, el marco podría resultar incomprendido y, como mencionamos, los participantes no siempre lo reconocen como un marco de broma ${ }^{21}$. Analizamos a continuación otras pistas o claves de contextualización (en el sentido de Gumperz 1982) que, junto con el uso de la metáfora y el anclaje deíctico (mediante el uso de clasificadores deícticos, pronombres demostrativos y personales, y género gramatical), contribuyen a construir el marco de las bromas sexuales entre los qom. Se trata de recursos indexicales (no referenciales), que dependen fuertemente del contexto en el que ocurren y cuya ejecución e interpretación adecuada se basa en los conocimientos culturales compartidos.

\section{FóRMULAS DE TRATAMIENTO}

El uso de apelativos, como izic 'amigo', que abre la interacción en el ejemplo (1), funciona como índice del género de broma o nayakyaGak: «cuando escucha izik, ya sabe que está jugando, haciendo broma», comenta uno de nuestros colaboradores qom. En este sentido, el uso del vocativo como fórmula de tratamiento, indexa relaciones sociales a la vez que construye y habilita el marco de la broma.

\section{CONTEXTO PARALINGÜÍSTICO}

Constituyen índices paralingüísticos el lugar, el tiempo (el momento del día), la edad y los instrumentos de caza. De esta manera, si este tipo de bromas sexuales se realiza en contextos urbanos o periurbanos, en los que las prácticas de marisca son infrecuentes, o bien entre jóvenes con escasa experiencia en la caza, o también en momentos del día inapropiados para estas prácticas, en estos casos el sentido literal de estas emisiones queda pragmáticamente anulado y la interpretación metafórica constituye la única opción. Se trata de elementos contextuales que permiten también enmarcar la broma.

21 Una observación similar es realizada por De Vienne, en tanto propone que, entre los trumai del Alto Xingú, la intención de las bromas es justamente desestabilizar al otro; $y$, con este fin, «they mobilize a more complex frame, also mentioned (but only in passing) by Bateson (1972: 141), which is constructed not upon the premise 'This is play' but rather around the question 'Is this play?'» (2012: 165).

\section{CANCELACIÓN DEL MARCO LITERAL}

El marco de la broma puede ser construido, así como también negociado $y$, en este sentido, podemos observar las negociaciones entre los enunciados y las fuerzas ilocucionarias. En algunos contextos, puede haber una respuesta que cancele el marco literal y confirme la ejecución del marco de juego. En estos casos, luego de un primer momento serio, la respuesta desencadena la risa. Observemos algunos ejemplos, en los cuales el interlocutor competente realiza un comentario que cancela la interpretación literal y confirma que se trata de una broma, en un marco lúdico:

\begin{tabular}{|c|c|c|c|c|}
\hline - ̃̃i-?epe & & i-layke & \multicolumn{2}{|c|}{ qageta-lyale } \\
\hline 1M-cazar & & 1A-buscar & \multicolumn{2}{|c|}{ oveja-DIM.F } \\
\hline \multicolumn{5}{|c|}{ 'Me voy a cazar, voy a buscar una ovejita' } \\
\hline -wotayke & $d a$ & ?an-?epe qalqa?a & $a-k a$ & $\begin{array}{l}\text { Pad- } \\
\text { owanaqte }\end{array}$ \\
\hline 3.querer & $\mathrm{NX}$ & 2A-cazar COORD & F-D:aus & 3pos-arma \\
\hline
\end{tabular}

-zi-shetayke

l-apat

11-desear/querer

3POS-carne

'Quiero [comer] carne'

$\begin{array}{lll}\text {-datoGoik } & \text { qalqo? } & \text { tokchigi } \\ \text { cocido } & \text { COORD } & \text { crudo }\end{array}$

'¿Cocida o cruda?'

Otras claves para construir el marco, como pueden ser las pautas prosódicas especiales de tempo, acento y tono, los gestos, los guiños, la postura corporal y las sonrisas necesitan ser aun estudiadas ${ }^{22}$.

Es la competencia comunicativa (lingüística, sociocultural y poética) de los hablantes -el bromista y su interlocutor o audiencia- la que les permite comunicarse según los modos socialmente apropiados: para que la audiencia capte el chiste es necesario que haya conocimiento compartido. Así también, se destaca el poder del humor, como observan Carty y Musharbash (2008: 215) en tanto facilita la comprensión, crea significados, e incluso crea los propios contextos a través de los cuales se hacen posibles los significados compartidos.

22 La imposibilidad de las autoras de ser parte de la audiencia -por ser mujeres y doqshe ('no indígenas')- limitó la participación en contextos espontáneos de broma. 


\section{PALABRAS FINALES. APROXIMACIÓN A LA FUNCIONALIDAD DE LAS BROMAS}

El análisis aquí propuesto nos permitió observar las construcciones metafóricas que se encuentran en uso en las bromas sexuales qom y mostró los vínculos entre el campo semántico de la marisca y el de la sexualidad. En una única expresión que no es anticipada ni explicada comunicativamente, se muestra de manera clara la estrecha asociación entre la sexualidad, la alimentación y las prácticas masculinas de subsistencia.

El trabajo constituye un punto de partida para comprender la naturaleza del humor y revisar las definiciones de humor que han sido típicamente focalizadas en la interpretación de la audiencia y en la aparición de la risa como elemento constitutivo. Así también, desde una perspectiva antropológica, el humor sexual permite pensar las nociones de erotismo entre los qom del Gran Chaco. Si bien la textualidad de una broma es reducida, constituye una estrategia discursiva condensadora en la cual las cuestiones interaccionales latentes pueden resultar sustanciales. En tanto evento discursivo complejo, el humor cubre diferentes funciones, más allá de hacer reír ${ }^{23}$. Nos preguntamos entonces cuáles son las funciones de las bromas qom que analizamos, tanto desde el punto de vista comunicativo como social.

En primer lugar, la alusión a símbolos compartidos da la posibilidad de expresar ciertos significados que de otra manera no podrían transmitirse. Condensando de manera extrema la relación entre lo dicho y lo no dicho, las bromas analizadas mostraron la clara elección de un lenguaje no directo para hablar del deseo y del sexo, del cuerpo y de las relaciones entre los géneros. En ellas, la metáfora posee no solo una función cognitiva, en tanto herramienta para hablar, conocer y actuar en relación con el erotismo, sino fundamentalmente social. Si en la vida cotidiana de los tobas (y de otros grupos chaqueños), la afectividad entre el hombre y la mujer no es explícitamente demostrada, el recurso de la indirección permite a los hombres hablar de un tópico del cual no suele hablarse, tal como mostró Black entre los zulu-hablantes de Sudáfrica ${ }^{24}$. A su vez, el humor presente en las bromas habilita, como ya sostuvo Beeman (2000), que las personas actúen las emociones dentro del espacio conceptualmente seguro de un marco discursivo lúdico. "El amor no debe mostrarse», expresa Chase-Sardi refiriéndose a las parejas nivaclé, «debe ser secreto, oculto. Dar las desvergonzadas expresiones públicas de cariño [...] es muestra de lujuriante hambre de sexo, es asemejarse a los animales» (1992: 40).

A su vez, el bromista muestra que comparte con la audiencia una idea en común sobre lo que es gracioso, y esto sirve para mantener o crear solidaridad entre compañeros (izik), lo que permite observar una dimensión de las bromas especialmente ligada a la regulación de las relaciones intersubjetivas (cf. Carty y Musharbash 2008; Rosengren 2010; Black 2012; De Vienne 2012). En este sentido, el humor puede servir para fortalecer o reafirmar normas y valores, y hacer explícita la pertenencia a un grupo: quienes bromean e interpretan la broma son aquellos que son miembros de un mismo colectivo (cf. Hay 1995).

Sin duda la descripción y comprensión de este tipo de discursos de humor, que no es independiente de la vida social y cultural entre los qom, permite un acercamiento a las nociones de erotismo y sexualidad, en tanto las bromas qom indexan prácticas y valores culturales a través del lenguaje figurado $y$, al mismo tiempo, indexan una estrategia interaccional particular. Por lo tanto, reflejan e instauran de una manera creativa las relaciones entre lenguaje, cultura y sociedad, y los individuos.

Abreviaturas: A (participante agente/activo); aus (ausente); COORD (coordinante); D (clasificador deíctico); DEM (demostrativo); DIM (diminutivo); DIR (direccional); dist (distante); DUR (aspecto durativo); EX (existencial); F (femenino); I (participante inactivo); INS (instrumental); INTERJ (interjección); INTERR (interrogativo); LOC (locativo); M (participante medio); NMZ (nominalizador); NX (nexo); POS (poseedor); PROG (aspecto progresivo); PRON (pronombre personal); VBZ (verbalizador); vert (vertical); 1 (primera persona); 2 (segunda persona); 3 (tercera persona), 4 (persona indefinida).

23 Cf. Hay (2000) para un relevamiento sociopragmático y una tipología de las funciones del discurso humorístico, con especial referencia a los estudios sobre el inglés.

24 El humor permite así lidiar o enfrentarse con ciertos temas, tales como la enfermedad o la muerte, o en nuestro caso,

la sexualidad. De hecho, entre los tipos de humor que identifica Hay (1995: 78), se distingue el humor sexual (humor sobre sexo como tópico) como aquel que se caracteriza porque los hablantes rompen algún tipo de tabú. 


\begin{tabular}{|c|c|c|c|c|c|c|c|c|c|c|c|c|}
\hline & \multicolumn{2}{|c|}{ labial } & \multicolumn{2}{|c|}{ alveolar } & \multicolumn{2}{|c|}{ palatal } & \multicolumn{2}{|c|}{ velar } & \multicolumn{2}{|c|}{ uvular } & \multicolumn{2}{|c|}{ glotal } \\
\hline oclusiva & $p$ & & $\mathrm{t}$ & $d$ & $\begin{array}{c}\text { tf } \\
{[\mathrm{ch}]}\end{array}$ & & $\mathrm{k}$ & g & $q$ & G & $?$ & \\
\hline fricativa & & & $\mathrm{s}$ & & $\int_{[\mathrm{sh}]}$ & $\begin{array}{c}3 \\
{[z]}\end{array}$ & & & & & & \\
\hline nasal & & $\mathrm{m}$ & & $\mathrm{n}$ & & $\underset{[\tilde{n}]}{n}$ & & & & & & \\
\hline tap & & & & $\begin{array}{c}\mathrm{r} \\
{[\mathrm{r} / \mathrm{d}]}\end{array}$ & & & & & & & & \\
\hline lateral & & & & 1 & & $\hat{\Lambda}$ & & & & & & \\
\hline glide & & w & & & & $y$ & & & & & & \\
\hline
\end{tabular}

\begin{tabular}{|c|c|c|}
\hline \multicolumn{3}{|c|}{ vocales } \\
\hline $\mathrm{i}$ & & $\mathrm{o}$ \\
\hline $\mathrm{e}$ & & \\
\hline & $\mathrm{a}$ & \\
\hline
\end{tabular}

TABLA 2.- Inventario fonológico [y ortográfico] toba.

En la Tabla 2 se presenta el inventario fonológico toba (en base a Messineo 2003: 36) y las correspondencias ortográficas [entre corchetes] que se utilizan en este trabajo.

\section{BIBLIOGRAFÍA CITADA}

Anderson, John y Edward Keenan. 1985. "Deixis», en Timothy Shopen (ed.), Language Typology and Syntactic Description: Grammatical Categories and the Lexicon: 259-308. Cambridge: Cambridge University Press.

Attardo, Salvatore. 1997. "The semantic foundations of cognitive theories of humor». Humor: International Journal of Humor Research 10 (4): 395-420. doi: <https://doi. org/10.1515/humr.1997.10.4.395>.

Balducci, María Isabel. 1982. Códigos de comunicación con el mundo animal entre los Toba-Taksik. Facultad de Filosofía y Letras, Universidad de Buenos Aires.

Basso, Keith. 1979. Portraits of the "Whiteman": Linguistic Play and Cultural Symbols among the Wetern Apache. New York: Cambridge University Press.

Bateson, Gregory. 1972. "A Theory of Play and Fantasy». Steps to an Ecology of Mind: 167-193. San Francisco: Chandler.

Bauman, Richard. 2002 [1975]. «El arte verbal como ejecución», en L. Golluscio, C. Courtis, C. Messineo, C. Oxman y S. Skura. (comps.), La Etnografía del Habla. Textos fundacionales. Buenos Aires: EUDEBA.

Beeman, William. 2000. "Humor», en Alessandro Duranti (ed.), Linguistic Lexicon for the Millennium. Número especial de Journal of Linguistic Anthropology 9(1-2): 103-106.
Black, Stephen. 2012. «Laughing to Death: Joking as Support Amid Stigma for Zulu-Speaking South Africans Living with HIV». Journal of Linguistic Anthropology 22(1): 87-108. doi: <https://doi.org/10.1111/j.1548-1395.2012.01140.x>.

Braunstein, José. 1983. «Algunos rasgos de la organización social de los indígenas del Gran Chaco». Trabajos de Etnología 2: 9-102.

Carty, John y Yasmine Musharbash. 2008. "You've Got to Be Joking: Asserting the Analytical Value of Humour and Laughter in Contemporary Anthropology». Anthropological Forum 18(3): 209-217. doi: <https://doi. org/10.1080/00664670802429347>.

Chase-Sardi, Miguel. 1992. "Pequeño decamerón nivaclé», en M. Chase-Sardi, A. Siffredi, y E. Cordeu (eds.), El gateo de los nuestros: Narrativa erótica indígena del Gran Chaco: 33-94. Buenos Aires: Del Sol.

Chase-Sardi, Miguel, Alejandra Siffredi y Edgardo Cordeu (eds.). 1992. El gateo de los nuestros: Narrativa erótica indígena del Gran Chaco. Buenos Aires: Del Sol.

Cúneo, Paola. 2013. Formación de palabras y clasificación nominal en el léxico etnobiológico en toba (guaycurú). Lincom Studies in Native American Linguistics 68. Múnich: Lincom Europa.

De los Ríos, Miguel. 1978-1979. «La expresión de la afección amorosa en la etnia mataco». Scripta Ethnologica 5: 27-54

De Vienne, Emmanuel. 2012. “'Make Yourself Uncomfortable.' Joking Relationships as Predictable Uncertainty Amog the Trumai of Central Brazil». HAU: Journal of Ethnographic Theory 2(2): 163-187. doi: <https://doi.org/10.14318/ hau2.2.010>.

Goffman, Erving. 1974. Frame Analysis: An essay on the organization of experience. Boston: Northeastern University Press. 
Gordillo, Gastón. 2005. Nosotros vamos a estar acá para siempre: Historias tobas. Buenos Aires: Biblos.

Gregor, Thomas. 1985. Anxious pleasures. The sexual lives of an Amazonian people. Chicaco: Chicaco University Press.

Gregor, Thomas y Donald Tuzin. 2001. Gender in Amazonia and Melanesia. An Exploration of the Comparative Mmethod. Berkeley: University of California Press.

Gumperz, John. 1982. Discourse Strategies. Cambridge: Cambridge University Press

Hay, Jennifer. 1995. Gender and Humour: Beyond a Joke. Tesis de Maestría. Victoria University of Wellington.

Hay, Jennifer. 2000. «Functions of Humor in the Conversations of Men and Women». Journal of Pragmatics 32: 709-42. doi: <https://doi.org/10.1016/s0378-2166(99)00069-7>.

Hugh-Jones, Christine. 1979. From the Milk River. Spatial and Temporal Processes in Northwest Amazonia. Cambridge: Cambridge University Press.

Idoyaga Molina, Amalia. 1976. "Matrimonio y pasión amorosa entre los mataco». Scripta Ethnologica 4(1): 6-67.

INDEC. 2010. Censo 2010 Argentina. Buenos Aires: Instituto Nacional de Estadísticas y Censos. <https://www.indec. gob.ar/indec/web/Nivel4-Tema-2-41-135>

Jolly, Margaret. 2001. "Damming the Rivers of Milk? Fertility, Sexuality, and Modernity in Melanesia and Amazonia», en Thomas A. Gregor y Donald Tuzin (eds.), Gender in Amazonia and Melanesia. An Exploration of the Comparative Method: 175-206. Berkeley: University of California Press.

Lagrou, Els. 2006. «Rir do poder e poder do riso nas narrativas e performances kaxinawa». Revista de Antropologia 49(1): 55-90. doi: <https://doi.org/10.1590/s0034$77012006000100003>$.

Lakoff, George y Mark Johnson. 1980. Metaphors We Live By. Chicago: Chicago University Press.

Lévi-Strauss, Claude. 1964. Mitológicas. México: Fondo de Cultura Económica.

Mauss, Marcel. 1926. "Parentés à plaisanteries». Annuaire de l'École pratique des hautes études, Section des sciences religieuses, Texte d'une communication présentée à I'Institut français d'anthropologie. París

Medrano, Celeste. 2013. Zoo-sociología qom: de cómo los tobas y los animales trazan sus relaciones en el Gran Chaco. Tesis doctoral. Universidad de Buenos Aires.

Messineo, Cristina. 2003. Lengua Toba (guaycurú). Aspectos gramaticales y discursivos. Münich: Lincom Europa Academic Publisher.

Messineo, Cristina. 2014. Arte verbal qom. Consejos, rogativas y relatos de El Espinillo (Chaco). Buenos Aires: Rumbo Sur.

Moreau, Reginald Ernest. 1941. «The Joking Relationship (Utani) in Tanganyika». Tanganyika Rotes and Records 12: $1-10$.
Moreau, Reginald Ernest. 1944. "The Joking Relationship in Tanganyika». Africa 14(3): 386-400. doi: <https://doi. org/10.2307/1157006>.

Morreall, John. 1987. The Philosophy of Laughter and Humor. Albany: State University of New York Press.

Morreall, John. 1989. «Enjoying Incongruity». Humor 2(1): 1-18. doi: <https://doi.org/10.1515/humr.1989.2.1.1>.

Mühlhäusler, Peter. 2012. «Prologue», en Anna Idström y Elizabeth Piirainen (eds.), Endangered Metaphors: 1-13. Amsterdam/Philadelphia: John Benjamins.

Otaegui, Alfonso. 2014. Les chants de nostalgie et de tristesse des Ayoreo du Chaco boréal paraguayen (une ethnographie de lien coupé). Tesis doctoral. École des Hautes Études en Sciences Sociales, Paris.

Otaegui, Alfonso. 2016. "“I'm Crying for the Beautiful Skin of the Jaguar." Laments, Non-Humans and Conviviality among the Ayoreo of the Northern Chaco", en J. Rivera (ed.), Amerindian Rites and Entities in South America: Reassessments and Comparisons. Nueva York/Oxford: Berghaghn Books.

Overing, Joanna. 2000. "The Efficacy of Laughter. The Ludic Side of Magic within Amazonian Sociality», en J. Overing y A. Passes (eds.), The Anthropology of Love and Anger. The Aaesthetics of Conviviality in Native Amazonia: 64-81. Londres/Nueva York: Routledge.

Paulme, Denise. 1939. "Parenté à plaisanteries et alliance par le sang en Afrique occidentale», Africa 12(4): 433-444. doi: <https://doi.org/10.2307/1155951>.

Radcliffe-Brown, A. R. 1940. «On Joking Relationships». Africa 8: 195-210. doi: <https://doi.org/10.2307/1156093>.

Radcliffe-Brown, A. R. 1949. "A Further Note on Joking Relationships». Africa 14: 133-140. doi: <https://doi.org/ 10.2307/1156517>

Rosengren, Dan. 2010. «Seriously Laughing: On Paradoxes of Absurdity among Matsigenka People», Ethnos 75(1): 102121. doi: <https://doi.org/10.1080/00141840903402468>.

Sherzer, Joel. 1985. "Puns and Jokes», en T. Van Dijk (ed.), Handbook of Discourse Analysis, 3(15). Londres: Academic Press Inc.

Sherzer, Joel. 1992 [1983]. Formas del habla Kuna. Una perspectiva etnográfica. Quito: ABYA-YALA.

Sherzer, Joel. 2002. Speech Play and Verbal Art. Austin: Universiy of Texas Press.

Siffredi, Alejandra y Edgardo Cordeu. 1992. "Prólogo», en M. Chase-Sardi, A. Siffredi, y E. Cordeu (eds.), El gateo de los nuestros: Narrativa erótica indígena del Gran Chaco: 7-31. Buenos Aires: Del Sol.

Tola, Florencia. 2004. 'Je ne suis pas seul(ement) dans mon corps'. Corps et multiplicités chez les Toba (Qom) du Chaco argentin. Tesis doctoral. École des Hautes Études en Sciences Sociales/ Universidad de Buenos Aires.

Tola, Florencia y Paola Cúneo. 2014. «Jeter ses filets et manipuler les c'urs. Érotisme et humour sexuel chez les Toba 
(Gran Chaco)», Conferencia presentada en el Séminaire d'Anthropologie Américaniste, Laboratoire d'Anthropologie Sociale del Collège de France.

Viveiros de Castro, Eduardo. 1992. From the Enemy Point of View. Humanity and Divinity in an Amazonian Society. Chicago: University of Chicago Press.
Wilbert, Johannes y Karin Simoneau. 1992. Folk Literature of South American Indians: General Index. Los Angeles: UCLA Latin American Center Publications, University of California.

Wright, Pablo. 2008. Ser-en-el-sueño: crónicas de historia y vida toba. Buenos Aires: Biblios. 